\title{
Risk factors of surgical site infections in hip hemiarthroplasty: a single-institution experience over nine years
}

\author{
Adrian Cheng Kiang $\underline{\operatorname{Lau}}^{1}$, MBBS, FRCSE, Ghim Hoe $\underline{\mathrm{NeO}}^{1}$, MD, Haw Chou $\underline{\mathrm{Le}}^{1}$, MBBS, FRCSE
}

\begin{abstract}
INTRODUCTION This study aims to describe the factors associated with surgical site infection (SSI) in elderly patients who underwent hip hemiarthroplasty following a hip fracture, in a single institution over a nine-year period. METHODS All patients who underwent hip hemiarthroplasty between 1 January 2004 and 31 December 2012 in our hospital were included in the present study. The detection of SSI was carried out by a trained, independent infection control nurse using the United States Centers for Disease Control and Prevention criteria. Demographic and clinical data were collected retrospectively. Demographics and clinical factors were analysed for potential associations with SSI. RESULTS Among the 1,320 patients who met the study inclusion criteria, a total 57 SSIs were documented, giving an infection rate of $4.3 \%$. Patients who waited for more than one week for surgery had a statistically significantly higher risk of SSI (odds ratio $3.030,95 \%$ confidence interval $1.075-8.545, p=0.036)$. The presence of SSI was also significantly associated with increased length of hospital stay $(p<0.001)$. The two main microorganisms detected were methicillin-resistant Staphylococcus aureus and Pseudomonas aeruginosa, which accounted for $50.9 \%$ and $26.3 \%$ of the SSIs, respectively.

CONCLUSION We concluded that the number of days from admission to surgery is a main risk factor for the development of SSI. Steps should, therefore, be taken to prevent unnecessary delay of surgery in elderly patients requiring hip hemiarthroplasty.
\end{abstract}

Keywords: hemiarthroplasty, neck of femur, surgical site infections

\section{INTRODUCTION}

Surgical site infection (SSI) is one of the greatest challenges faced by an orthopaedic surgeon. In 2002, Whitehouse et al reported that orthopaedic SSI prolonged the median length of hospital stay by two weeks per patient, doubled rehospitalisation rates and increased healthcare costs by more than $300 \%$. ${ }^{(1)}$ Klevens et al estimated that $20 \%$ of all healthcare-associated infections are SSIs, the second most common type of hospital-acquired infection in the United States. ${ }^{(2)}$

SSIs become even more problematic when they involve elderly patients who had just undergone hemiarthroplasty for osteoporotic hip fractures. As elderly patients generally have poorer physiological reserves, additional insults in the form of multiple debridement surgeries and infection tend to lead to high mortality rates. The relatively avascular acetabular labrum and cartilage, as well as the ease of biofilm formation on prosthesis, further contributes to the resistant nature of deep-seated infections. Therefore, many centres around the world have endeavoured to find the risk factors associated with SSI. To the best of our knowledge and based on our literature search, no study to date has been done to describe the risk factors associated with SSI after hip hemiarthroplasty in an Asian population. This study aimed to report the incidence of SSI in elderly patients who had undergone hip hemiarthroplasty in a single training institution over a nine-year period and identify the significant risk factors associated with SSI in our patient population.

\section{METHODS}

All patients who underwent primary hip hemiarthroplasty in Changi General Hospital (CGH), Singapore, between 1 January 2004 and 31 December 2012 were included in the present study. Hip fracture patients who underwent total hip replacement or revision bipolar hemiarthroplasty were excluded. The surgeries were performed by consultants, associate consultants or registrars from the same institution. Surgeries that were performed by registrars were supervised by a qualified surgeon. The detection of SSI in the patients was carried out by a trained, independent infection control nurse. All case notes regarding patients who underwent primary hip hemiarthroplasty during the aforementioned period were reviewed at one month and at one year after surgery; any documented SSI that met the United States Centers for Disease Control and Prevention (CDC) criteria $^{(3)}$ was noted.

The CDC, which has defined a set of criteria for the purpose of surveillance, with the ultimate aim of reducing SSI risk, categorises SSIs into: (a) superficial incisional; (b) deep incisional; and (c) organ/space SSIs. Superficial SSI, defined as infection that occurs within 30 days after a procedure, involves only the skin and subcutaneous tissue of the incision, and has at least one of the following characteristics: purulent discharge from the superficial incision; positive culture from an aseptically obtained culture of fluid; clinical signs of tenderness; or localised swelling, redness or warmth. Deep incisional SSI is defined as infection that occurs within 90 days of a procedure, and involves the fascial and muscle layers. In the present study, organ/space SSI refers to infection involving the bone or hip joint.

To allow for comparison of infection rates between institutions and analysis of SSI rates within an institution over time, the CDC has developed the National Nosocomial Infections Surveillance (NNIS) risk index system ${ }^{(4)}$ to enable hospitals report cumulative

${ }_{1}^{1}$ Department of Orthopaedic Surgery, Changi General Hospital, Singapore

Correspondence: Dr Adrian Lau Cheng Kiang, Associate Consultant, Department of Orthopaedic Surgery, Changi General Hospital, 2 Simei Street 3 , Singapore 529889. adrianlck@yahoo.com 
wound infection data. The NNIS risk index ranges from 0 to 3 points. One point is added to the patient's risk index for any of the following three variables: (a) an operation that is contaminated or dirty; (b) a patient with an American Society of Anesthesiologists (ASA) preoperative score of 3, 4 or 5; and (c) the duration of the operation exceeds the 75th percentile (determined using the NNIS database). In the present study, the maximum NNIS risk index was 2, as all hemiarthroplasty procedures are considered clean operations.

The demographic and clinical data of all the patients included in the study were retrospectively collected from the digital database available within the hospital. The collected data included the patient's age, gender, ASA and NNIS scores, days to surgery (defined as the number of days from admission to surgery), duration of hospital stay (defined as number of days from hospital admission to hospital discharge), time at which surgery was carried out (before noon, noon to 5 pm or after 5 pm), duration of surgery, type of anaesthesia used, method of skin closure, and seniority of the main operating surgeon. The presence/absence of diabetes mellitus, perioperative serum haemoglobin, creatinine and albumin levels, and the presence of aspirin, warfarin and steroids in the discharge medications, were also recorded. For cases with documented SSI, the type of SSI (based on CDC criteria) was recorded. The microorganisms involved in the SSI, based on available cultures, were also noted.

All statistical analyses were done using the Statistical Package for the Social Sciences version 16.0 (SPSS Inc, Chicago, IL, USA). All tests of hypotheses were two-tailed and statistical significance testing was performed at the 0.05 level. Variables were summarised according to infection status (i.e. SSI: yes/no). Chi-square test was used to compare categorical variables (e.g. ASA score, NNIS score, seniority of the main operating surgeon) across infection statuses. Mean values of continuous variables (e.g. age and duration of hospital stay) were compared between infection statuses using the two-sample $t$-test. Finally, a multivariate logistic regression model was fitted; the model included the number of days to surgery, NNIS score, method of skin closure, serum albumin level and the presence/absence of diabetes mellitus.

\section{RESULTS}

A total of 1,320 patients (mean age $78.6 \pm 8.8$ years) were recruited for the present study. These patients had undergone hemiarthroplasty for femoral neck fractures. Of these, $1,002(75.9 \%)$ were women (mean age $76.8 \pm 8.8$ years) and $318(24.1 \%)$ were men (mean age $79.1 \pm 8.7$ years), with a significantly older male population $(p<0.001)$. The median number of days to surgery was 4 (interquartile range $3-7$ ) days and the mean duration of surgery was $78.6 \pm 25.4 \mathrm{~min}$.

Among the 1,320 patients, a total of 57 SSIs were documented, giving an overall infection rate of $4.3 \%$. Of these 57 SSIs, 34 were superficial incisional SSIs, 3 were deep incisional SSIs and 20 were organ/space SSIs. Among the 34 superficial incisional SSIs, methicillin-resistant Staphylococcus aureus (MRSA) was isolated in $14(41.2 \%)$ cases, Pseudomonas aeruginosa was isolated in
$10(29.4 \%)$ cases, no organisms were isolated in 3 (8.8\%) cases and no cultures were performed in $7(20.6 \%)$ cases. Among the 23 deep incisional and organ/space SSIs, MRSA was isolated in 15 (65.2\%) cases, while Pseudomonas aeruginosa was isolated in $5(21.7 \%)$ cases. MRSA and Pseudomonas aeruginosa were the two major organisms involved in the SSIs of hemiarthroplasty patients. MRSA was involved in $50.9 \%$ of all cases, and Pseudomonas aeruginosa, in $26.3 \%$ of all cases.

The results of the univariate analysis are shown in Table I. While age and gender were not found to be associated with infection ( $p=0.285$ and $p=0.301$, respectively), a significant association between days to surgery and infection was observed. Patients who underwent the surgery within two days of admission had an infection rate of $2.3 \%$, whereas those who were underwent surgery between 3-7 days after admission had an infection rate of $4.3 \%$. The infection rate further increased to $6.8 \%$ in patients who were operated on after seven days of admission ( $p=0.026)$. Patients who received general anaesthesia had an infection rate of $6.3 \%$, which was slightly higher than the $4.0 \%$ infection rate of those who had received regional anaesthesia; this difference was, however, not statistically significant $(p=0.154)$. With regard to the method of skin closure, prolene closure had the highest rate of infection $(4.9 \%)$, while staples closure had an infection rate of $3.9 \%$ and monocryl closure had no documented SSI ( $p=0.034)$.

Although patients with a higher ASA score had a higher rate of infection, this difference was not statistically significant $(p=0.161)$. Similar results were found when analysing the NNIS score and its association with infection; although patients with a greater number of risk factors appeared to have a higher rate of infection, the difference was also not statistically significant $(p=0.053)$. Interestingly, there was no association between the duration of surgery and infection. Surgeries that took longer than $80 \mathrm{~min}$ had an SSI rate of $4.3 \%$ compared to $4.4 \%$ in surgeries lasting less than $80 \mathrm{~min}(\mathrm{p}=0.928)$. Infection rate was also not influenced by the time of the day (morning, afternoon or night) the surgery was performed $(p=0.705)$. Hemiarthroplasties performed by surgeons who were of consultant grade and above had an SSI rate of $3.4 \%$; this rate is lower than that of surgeries performed by surgeons who were associate consultants and below $(p=0.195)$.

Patients with diabetes mellitus had a higher SSI rate than those without diabetes mellitus $(5.6 \%$ vs. $3.7 \%, p=0.121)$. Neither a haemoglobin level $<10 \mathrm{~g} / \mathrm{dL}$ nor a serum creatinine level $>150 \mu \mathrm{mol} / \mathrm{L}$ was associated with an increased risk of SSI $(p=0.220$ and $p=0.207$, respectively). Although patients with a serum albumin level $\leq 20 \mathrm{~g} / \mathrm{L}$ had an SSI rate of $14.3 \%$ and those with a serum albumin level $>30 \mathrm{~g} / \mathrm{L}$ had an SSI rate of $5.0 \%$, this difference was not statistically significant $(p=0.056)$. In the present study, postoperative prescription of warfarin, aspirin or steroids was not significantly associated with increased SSI rates. However, the presence of SSI led to prolonged hospitalisation stay; compared to SSI-free patients, those with SSI had a longer mean hospitalisation stay $(18.9 \pm 12.5$ days vs. $38.8 \pm 39.1$ days, $p<0.001)$

The results of the multivariate analysis are shown in Table II. We found that patients who waited more than one week for surgery had a significantly higher risk of SSI (odds ratio 3.030, 95\% 
Table I. Results of the univariate analysis to detect associations between the factors studied and surgical site infections.

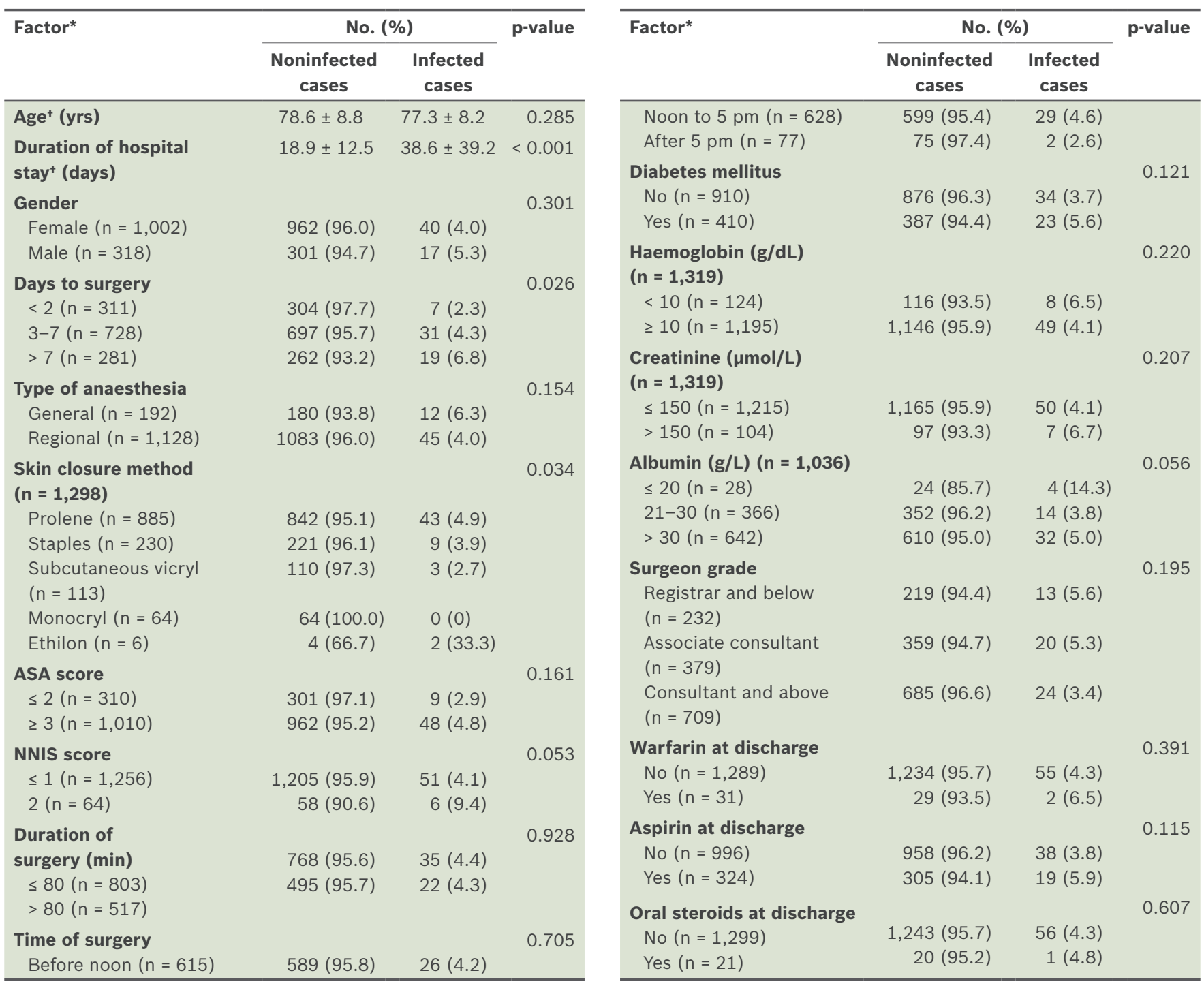

*Unless otherwise stated, the sample size is 1,320. +Continuous variables - age and duration of hospital stay - are presented as mean \pm standard deviation, and their statistical analysis was carried out using two-sample $t$-test. ASA: American Society of Anesthesiologists; NNIS: National Nosocomial Infections Surveillance

Table II. Results of the multivariate analysis.

\begin{tabular}{|c|c|c|c|}
\hline Factor & Odds ratio & $95 \% \mathrm{Cl}$ & p-value \\
\hline \multicolumn{4}{|c|}{ Days to surgery (ref: $0-2$ ) } \\
\hline $3-7$ & 2.210 & $0.839-5.822$ & 0.108 \\
\hline$>7$ & 3.030 & $1.075-8.545$ & 0.036 \\
\hline \multicolumn{4}{|c|}{ Diabetes mellitus (ref: No) } \\
\hline Yes & 1.204 & $0.660-2.196$ & 0.545 \\
\hline \multicolumn{4}{|c|}{ NNIS score (ref: $\leq 1$ ) } \\
\hline 2 & 1.747 & $0.582-5.239$ & 0.320 \\
\hline \multicolumn{4}{|c|}{ Albumin (ref: > $30 \mathrm{~g} / \mathrm{L}$ ) } \\
\hline$\leq 20$ & 3.073 & $0.964-9.800$ & 0.058 \\
\hline $21-30$ & 0.704 & $0.366-1.349$ & 0.289 \\
\hline \multicolumn{4}{|c|}{$\begin{array}{l}\text { Skin closure method* } \\
\text { (ref: vicryl) }\end{array}$} \\
\hline Prolene & $105,086,279$ & 0.000 & 0.997 \\
\hline Monocryl & 1.068 & 0.000 & 1.000 \\
\hline Ethilon & $479,707,636$ & 0.000 & 0.997 \\
\hline Staples & $85,133,062$ & 0.000 & 0.997 \\
\hline
\end{tabular}

* For skin closure method, no conclusion could be drawn and no data for $95 \% \mathrm{Cl}$ was generated due to the small number of patients in each subgroup. $\mathrm{Cl}$ : confidence interval; NNIS: National Nosocomial Infections Surveillance confidence interval $1.075-8.545, \mathrm{p}=0.036)$, after controlling for number of days to surgery, NNIS risk score, method of skin closure, serum albumin level and presence/absence of diabetes mellitus. No conclusion could be drawn regarding the association between the method of skin closure and risk of SSI infection due to the small number of patients in each subgroup.

\section{DISCUSSION}

In this retrospective study that involved information collected over nine years, the overall SSI infection rate was $4.3 \%$. This rate is similar to that reported by other researchers. For instance, Ridgeway et al reported an infection rate of $4.97 \%$ in a study involving 5,769 hemiarthroplasties of the hip. ${ }^{(5)}$ SSIs have been shown to be associated with longer hospital stays, increased costs and higher mortality. ${ }^{(6)}$ Indeed, the present study also found that patients with SSI had hospital stays that were more than twice that of patients without SSI.

Numerous studies have shown that delay in surgery for femoral neck fractures results in higher mortality. ${ }^{(7,8)}$ In a meta- 
analysis, Shiga et al showed that surgical delay of more than 48 hours increased the 30-day and 1-year mortality by $41 \%$ and $32 \%$, respectively. ${ }^{(9)}$ In the present study, we found that a delay in surgery was associated with an increased rate of SSI; this association remained significant even after controlling for other factors such as diabetes mellitus and NNIS score. Common reasons for delays in surgery include anaesthesia workup, cardiac assessment, the presence of anticoagulation and electrolyte imbalance. Our finding emphasises the importance of early surgery among elderly patients requiring hip hemiarthroplasties, so as to reduce the risk of SSI.

The present study also investigated the relationship between surgeon experience and the incidence of SSI. There was a trend toward a lower infection rate in hemiarthroplasties performed by surgeons who were consultants and above, although this was not statistically significant. Possible reasons for the lower SSI rate observed in more senior surgeons include better discipline in the operating theatre in terms of sterility and draping, more skilful handling of soft tissue and greater ability to preserve vascularity, leading to less blood loss.

Patients with diabetes mellitus are at increased risk of contracting SSI, as shown in a recent study by Everhart et al, which found that diabetes mellitus was an independent risk factor for SSI after total joint arthroplasty. ${ }^{(10)}$ This could be due to vascular insufficiency and hyperglycaemia-related impairment of the immune response. In the present study, we also found a higher SSI rate in patients with diabetes mellitus, although this did not reach statistical significance.

There is no accepted general consensus regarding the best method of skin closure after hip and knee arthroplasties. A 2010 meta-analysis by Smith et al found that the risk of SSI increased threefold when staples were used instead of sutures. ${ }^{(11)}$ In the present study, prolene closure, which resulted in the highest rate of infection, was the method of choice for most surgeons, accounting for $68.2 \%$ of all skin closures. Interestingly, no infection was noted in the group of patients who received monocryl closures. Monocryl is often used in subcuticular closures for better cosmetic appearance. Based the findings of the present study, monocryl may also have the added advantage of a lower infection rate.

MRSA and Pseudomonas aeruginosa were the two most common microorganisms implicated in the present study. MRSA accounted for $65.2 \%$ of deep incisional and organ/space SSIs, and $50.9 \%$ of all SSIs. Compared to the methicillin-sensitive strain of Staphylococcus aureus, MRSA has been associated with higher rates of patient morbidity and mortality. ${ }^{(12)}$ Between 1992 and 2003 , the prevalence of MRSA increased from $32 \%$ to $64 \%$ of all isolated nosocomial pathogens found on patients in hospital intensive care units. ${ }^{(13)}$ This increase in prevalence has led many hospitals, including ours, to carry out MRSA screening in patients undergoing surgery, so as to reduce the rate of MRSA colonisation as well as the incidence of MRSA-related SSI.

This study was not without limitations. As this is a retrospective study, it is particularly prone to information bias. Thus, misclassification of the factors being studied (such as diabetes mellitus) may occur due to insufficient documentation. Furthermore, although SSI trends were observed with regard to the type of anaesthesia used, the presence of diabetes mellitus, ASA score, NNIS score and serum albumin level $<20 \mathrm{~g} / \mathrm{L}$, our analysis did not reach statistical significance, possibly due to the insufficient sample sizes. In addition, the surveillance data for SSIs in the year 2012 was not available, which could have led to under-reporting of the infection rate.

In conclusion, a significant risk factor for SSI in hemiarthroplasty is the number of days to surgery. Therefore, it is recommended that surgery should be carried out as soon as possible in elderly patients who require hemiarthroplasty, in order to reduce the occurrence of SSI. Unnecessary postponement of surgery should be minimised.

\section{ACKNOWLEDGEMENTS}

We extend our thanks to Sister Li Jie and infection control nurses from $\mathrm{CGH}$ for their assistance in carrying out surveillance on the surgical site infections, and all the $\mathrm{CGH}$ orthopaedic surgeons who performed the aforementioned surgeries. We also thank Rachel Phillips, Biostatistician, Singapore Clinical Research Institute, for her help in data analysis.

\section{REFERENCES}

1. Whitehouse JD, Friedman ND, Kirkland KB, Richardson WJ, Sexton DJ. The impact of surgical-site infections following orthopedic surgery at a community hospital and a university hospital: adverse quality of life, excess length of stay, and extra cost. Infect Control Hosp Epidemiol 2002; 23:183-9.

2. Klevens RM, Edwards JR, Richards CL Jr, et al. Estimating health careassociated infections and deaths in U.S. hospitals, 2002. Public Health Rep 2007; 122:160-6.

3. Horan TC, Gaynes RP, Martone WJ, Jarvis WR, Emori TG. CDC definitions of nosocomial surgical site infections, 1992: a modification of CDC definitions of surgical wound infections. Infect Control Hosp Epidemiol 1992; 13:606-8.

4. Emori TG, Culver DH, Horan TC, et al. National nosocomial infections surveillance system (NNIS): description of surveillance methods. Am J Infect Control 1991; 19:19-35.

5. Ridgeway S, Wilson J, Charlet A, et al. Infection of the surgical site after arthroplasty of the hip. J Bone Joint Surg Br 2005; 87:844-50.

6. Lee J, Singletary R, Schmader K, et al. Surgical site infection in the elderly following orthopaedic surgery. Risk factors and outcomes. J Bone Joint Surg Am 2006; 88:1705-12.

7. Moran CG, Wenn RT, Sikand M, Taylor AM. Early mortality after hip fracture: is delay before surgery important? J Bone Joint Surg Am 2005; 87:483-9.

8. Zuckerman JD, Skovron ML, Koval KJ, Aharonoff G, Frankel VH. Postoperative complications and mortality associated with operative delay in older patients who have a fracture of the hip. J Bone Joint Surg Am 1995; 77:1551-6.

9. Shiga T, Wajima Z, Ohe Y. Is operative delay associated with increased mortality of hip fracture patients? Systematic review, meta-analysis, and meta-regression. Can J Anaesth 2008; 55:146-54.

10. Everhart JS, Altneu E, Calhoun JH. Medical comorbidities are independent preoperative risk factors for surgical infection after total joint arthroplasty. Clin Orthop Relat Res 2013; 471:3112-9.

11. Smith TO, Sexton D, Mann C, Donell S. Sutures versus staples for skin closure in orthopaedic surgery: meta-analysis. BMJ 2010; 340:c1199.

12. Melzer M, Eykyn SJ, Gransden WR, Chinn S. Is methicillin-resistant Staphylococcus aureus more virulent than methicillin-susceptible S. aureus? A comparative cohort study of British patients with nosocomial infection and bacteremia. Clin Infect Dis 2003; 37:1453-60.

13. Blumberg HM. Community acquired versus healthcare-associated methicillin resistant (MRSA) infections: is the distinction blurring? Clinical Updates in Infectious Diseases 2009; 12:1-6. 